\title{
Concurrent spinal schwannoma and meningioma mimicking a single cervical dumbbell-shaped tumor: case report
}

\author{
Takeshi Oichi, MD, ${ }^{1}$ Hirotaka Chikuda, MD, PhD, ${ }^{1}$ Teppei Morikawa, MD, PhD, ${ }^{2}$ \\ Harushi Mori, MD, PhD, ${ }^{3}$ Daisuke Kitamura, MD, ${ }^{1}$ Junya Higuchi, MD, ${ }^{1}$ Yuki Taniguchi, MD, PhD, ${ }^{1}$ \\ Yoshitaka Matsubayashi, MD, ${ }^{1}$ Yasushi Oshima, MD, PhD, ${ }^{1}$ and Sakae Tanaka, MD, PhD'
}

Departments of ${ }^{1}$ Orthopedic Surgery, ${ }^{2}$ Pathology, and ${ }^{3}$ Radiology, The University of Tokyo Hospital, Tokyo, Japan

\begin{abstract}
Dumbbell-shaped tumors consisting of 2 different tumors are extremely rare. Herein, the authors present a case of concurrent spinal schwannoma and meningioma mimicking a single cervical dumbbell-shaped tumor. A 64-year-old man presented with a 5-year history of gradually exacerbating left occipital pain without clinical evidence of neurofibromatosis. Magnetic resonance imaging showed an extradural tumor along the left C-2 nerve root with a small intradural component. The tumor was approached via a C-1 hemilaminectomy. The intradural tumor was resected together with the extradural tumor after opening the dura mater. The intradural tumor was attached to the dura mater around the exit point of the C-2 nerve root. Intraoperative biopsy revealed that the extradural tumor was a schwannoma and that the intradural tumor was a meningioma. The dura mater adjacent to the tumor was then coagulated and resected. Postoperative pathological examination confirmed the same diagnoses with no evidence of continuity between the intra- and extradural components. The patient's postoperative clinical course was uneventful. Clinicians should be aware that cervical dumbbell-shaped tumors can consist of 2 different tumors.
\end{abstract}

http://thejns.org/doi/abs/10.3171/2015.3.SPINE141315

KEY WORDS cervical spine; schwannoma; meningioma; pathology; oncology

$\mathrm{D}$ UMBBELL-SHAPED tumors are relatively common and account for $18 \%$ of spinal cord tumors. ${ }^{10} \mathrm{In}$ the cervical spine, $50 \%$ of all tumors are dumbbell shaped $^{4}$ and schwannoma is the most common cause. ${ }^{10}$ However, dumbbell-shaped tumors consisting of 2 different tumors are extremely rare. ${ }^{8}$ Herein, we present a case of concurrent extradural schwannoma and intradural meningioma mimicking a single cervical dumbbell-shaped tumor. Intraoperative biopsy from the extra- and intradural components was useful to determine the appropriate surgical treatment.

\section{Case Report}

History and Presentation

A 64-year-old man presented with a 5-year history of gradually exacerbating left occipital pain. Neurological examination did not reveal any neurological deficit, ex- cept for increased tendon reflexes in the right upper limb and both lower limbs. No subcutaneous nodules were observed, and the patient's vesicorectal function was normal. There was no family history of neurofibromatosis.

Magnetic resonance imaging showed a dumbbellshaped lesion involving the left C-2 nerve root and compressing the cervical spinal cord (Fig. 1). Gadoliniumenhanced T1-weighted MRI showed a thickened ventral dura mater (Fig. 1C), suggesting the presence of intradural tumor. However, preoperative MRI could not differentiate the intradural tumor from the extradural tumor. No other spinal or intracranial tumors were observed.

\section{Operative Procedure}

The tumor was approached via a C-1 hemilaminectomy. The extradural tumor was dissected just distal to its transition from the normal nerve root. Intraoperative ultrasonography showed an additional intradural component, 

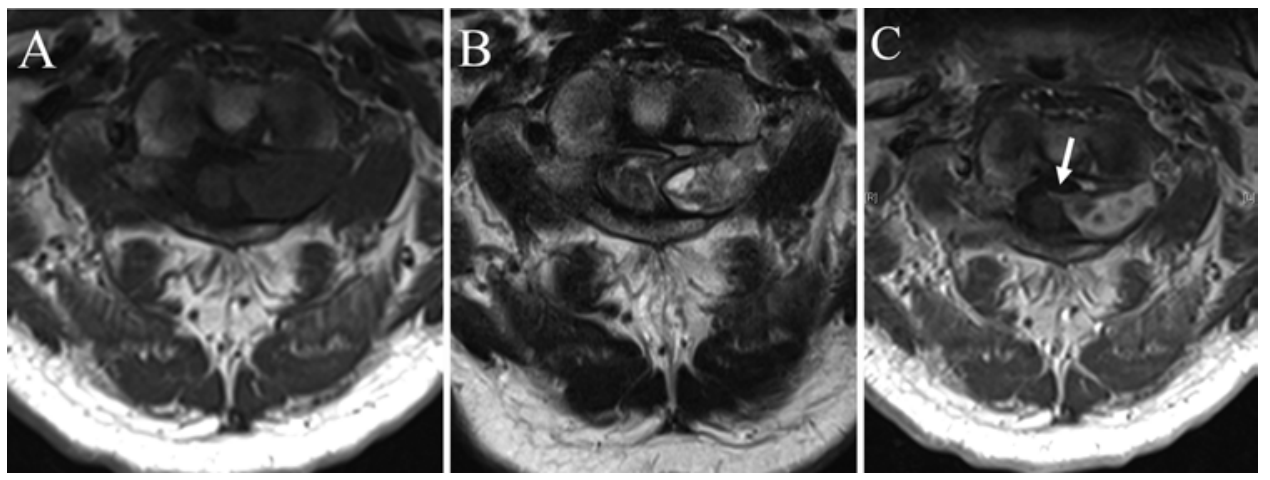

FIG. 1. Axial preoperative MR images showing an extradural tumor involving the left C-2 nerve root with a small intradural component. T1-weighted (A), T2-weighted (B), and gadolinium-enhanced T1-weighted (C) MR images could not definitively differentiate the extra- and intradural components. A thickened ventral dura mater is seen in panel $\mathrm{C}$ (arrow).

which was then inspected after opening the dura mater, revealing that it was firmly attached to the ventral dura mater around the $\mathrm{C}-2$ nerve root exit and did not appear to arise from the C-2 nerve root filaments (Fig. 2). The intradural tumor was resected together with the extradural tumor; there was no macroscopic continuity between the 2 tumors. The extradural tumor appeared as a homogeneous, soft, white-yellow, and encapsulated mass. The intradural tumor appeared as a homogeneous, firm, whiteyellow, and globular mass. The intraoperative pathological report showed that the extradural tumor was a schwannoma and the intradural tumor was a meningioma. We therefore coagulated and resected the adjacent dura mater. The dural defect was repaired using a 0.3-mm-thick expanded polytetrafluoroethylene (ePTFE) surgical membrane (GORE PRECLUDE MVP Dura Substitute, W. L. Gore \& Associates, Inc.).

\section{Pathological Findings}

The extradural tumor showed fascicular proliferation of spindle-shaped tumor cells with nuclear palisading (Fig. 3A). Cystic changes and hemosiderin deposits were occasionally observed. Both the dorsal nerve root ganglion and normal nerve root were observed at both ends. Immunohistochemically, the tumor cells were diffusely positive for S100 protein. Thus, the extradural tumor was diagnosed as schwannoma.

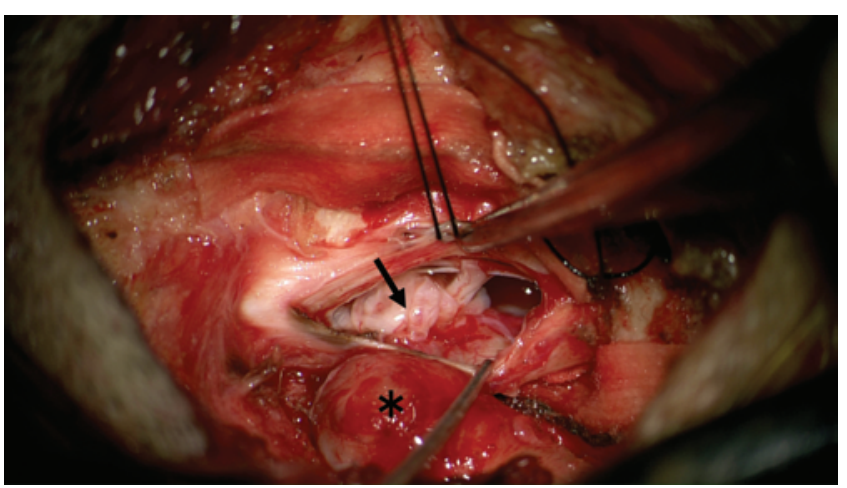

FIG. 2. Gross intraoperative findings showed the absence of macroscopic continuity between the intradural tumor (arrow) and the extradural tumor (asterisk). The intradural tumor was attached to the ventral dura mater around the left $\mathrm{C}-2$ nerve exit.
The specimens from the intradural component showed proliferation of oval-shaped tumor cells with scattered psammoma bodies (Fig. 3B). There were no schwannoma cells and tumor cell invasion of the nerve fibers. Immuno-

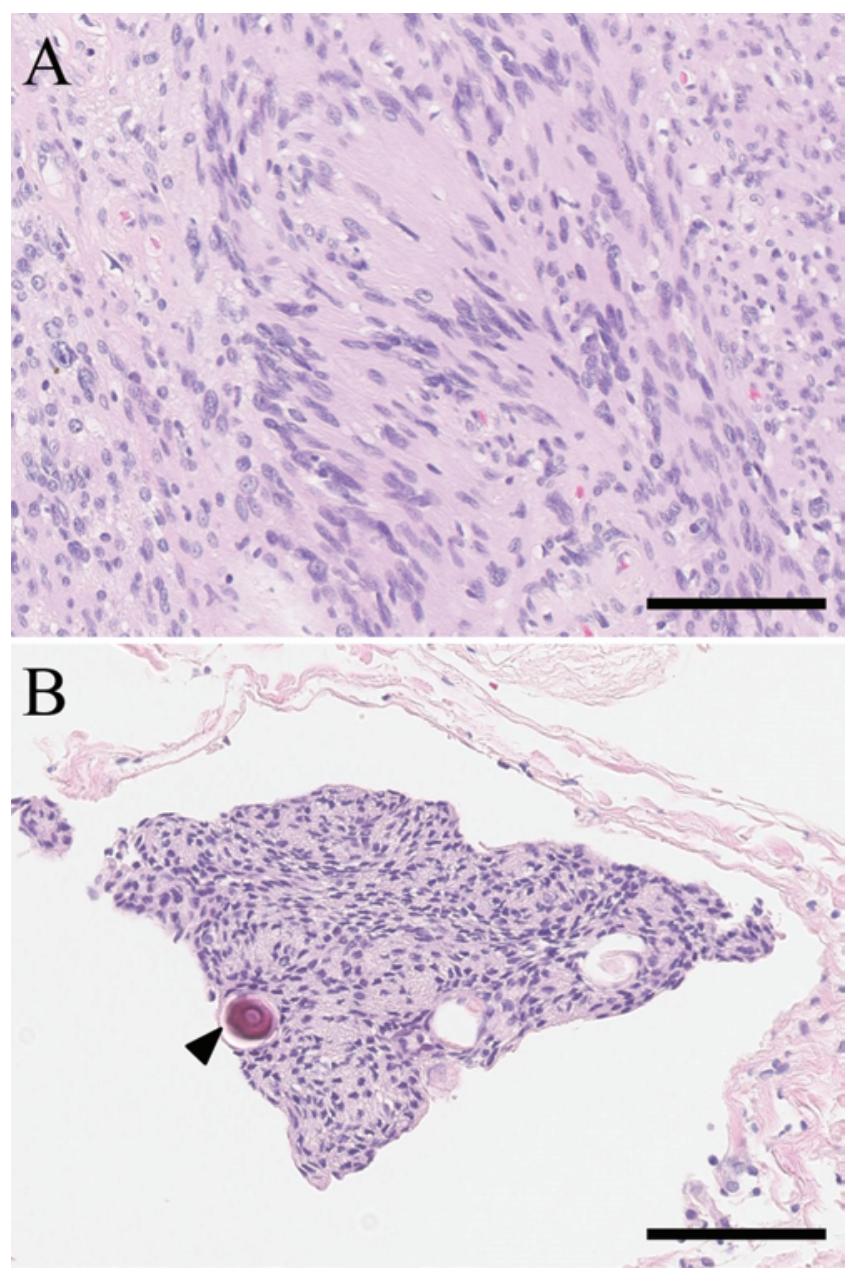

FIG. 3. Immunohistochemical analysis of the extra- and intradural lesions. A: The extradural tumor showed fascicular proliferation of spindle-shaped tumor cells with nuclear palisading. B: The intradural tumor showed proliferation of oval-shaped tumor cells with scattered psammoma bodies (arrowhead). H \& E, original magnification $\times 200$; bar $=100 \mu \mathrm{m}$. 
histochemically, the tumor cells were completely negative for S100 protein. Thus, the intradural tumor was diagnosed as meningioma.

The resected dura mater was free from tumor cells, and the pathological examinations did not show evidence of continuity between the extra- and intradural components.

\section{Postoperative Course}

The postoperative course was uneventful, and the patient's occipital pain improved immediately after surgery. Six months after surgery, the patient had no clinical symptoms related to the tumor, and MRI showed no tumor recurrence (Fig. 4).

\section{Discussion}

This case highlights 2 important issues. First, concurrent schwannoma and meningioma can occur at the same nerve root, mimicking a single cervical dumbbell-shaped tumor. Second, the intraoperative biopsy from both extraand intradural components was useful to determine the optimal surgical procedure.

The pathomechanism of concurrent schwannoma and meningioma occurring at a single nerve root is unclear. Several possible hypotheses have been postulated. The first is that the preexisting extradural schwannoma alters the microenvironment, facilitating the development of intradural meningioma. ${ }^{2,8}$ In relation to this hypothesis, we have to consider the differential diagnosis for reactive meningothelial hyperplasia that may be caused by a preexisting tumor, such as pilocytic astrocytoma. ${ }^{11,13,14}$ However, most cases of reactive meningothelial hyperplasia are detected only by microscopy and have multicentric growth patterns with discontinuous patches of hyperplastic nest. ${ }^{11}$ In contrast, in our case, the intradural tumor was a single tumor that was easily discernible macroscopically. Thus, we believe that the intradural tumor represents meningioma, but not meningothelial hyperplasia.

The second hypothesis is that 2 different tumors differentiate from common progenitor cells. ${ }^{1-3,6,7}$ Schwannomas are neuroectodermal tumors that are thought to arise from Schwann cells of dorsal nerve roots, whereas meningiomas are mesodermal tumors that are thought to arise from the arachnoid membrane. However, some authors have postulated that schwannoma and meningioma cells arise from the same mesenchymal cell. ${ }^{5,12}$ It is possible that the present case exhibited a multidirectional growth potential of progenitor cells. The third hypothesis is that 2 different tumors develop contiguously at the same place coincidentally ${ }^{3}$ because both schwannoma and meningioma are relatively common.

In the present case, intraoperative biopsy from both the extra- and intradural components provided critical information for appropriate surgical treatment. We presumed preoperatively that the tumor was a single schwannoma originating from the left C-2 nerve root and did not expect coexistence with another tumor. When the intraoperative biopsy revealed that the intradural tumor was a meningioma, we were able to add the surgical procedures of coagulating and resecting the dura mater to prevent recurrence. ${ }^{9}$ Thus, even if the tumor appears as a single dumbbellshaped tumor, intraoperative biopsy samples from both

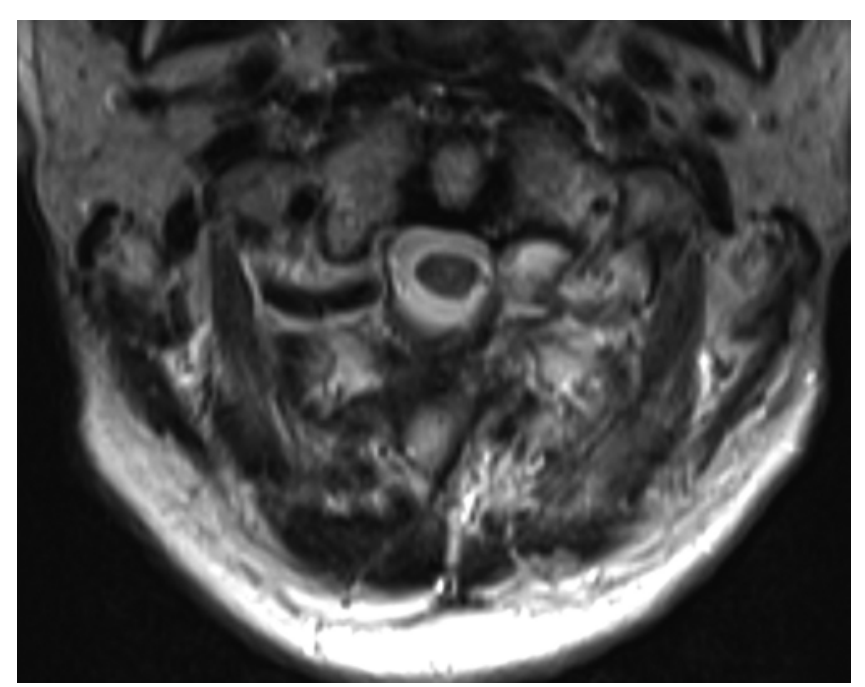

FIG. 4. Axial T2-weighted MR image obtained 6 months after surgery, showing decompression of the spinal cord. No residual tumor was noted.

extra- and intradural components should be analyzed to provide appropriate treatment, especially when continuity between the 2 components is ambiguous.

\section{Conclusions}

Concurrent schwannoma and meningioma can present as a single cervical dumbbell-shaped tumor. Intraoperative biopsy from both intra- and extradural components is useful for surgical decision making. We must be aware that a cervical dumbbell-shaped tumor can consist of 2 different tumors. Further reports should be accumulated to elucidate the pathogenesis of 2 different tumors occurring at the same nerve root.

\section{References}

1. Angiari P, Corradini L, Falasca A, Crisi G, Botticelli AR, Merli GA: Associated spinal meningioma and neurinoma. A case report. Acta Neurol (Napoli) 9:218-223, 1987

2. Brandwein-Gensler M, Urken M, Wang B: Collision tumor of the thyroid: a case report of metastatic liposarcoma plus papillary thyroid carcinoma. Head Neck 26:637-641, 2004

3. Dorizzi A, Crivelli G, Marra A, Scamoni C, Dario A, Bonfanti N, et al: Associated cervical schwannoma and dorsal meningioma. Case report and review of the literature. J Neurosurg Sci 36:173-176, 1992

4. el-Mahdy W, Kane PJ, Powell MP, Crockard HA: Spinal intradural tumours: Part I-Extramedullary. Br J Neurosurg 13:550-557, 1999

5. Feigin I: Mixed mesenchymal tumors: meningioma and nerve sheath tumor. J Neuropathol Exp Neurol 37:459-470, 1978

6. Kim DG, Paek SH, Chi JG, Chun YK, Han DH: Mixed tumour of schwannoma and meningioma components in a patient with NF-2. Acta Neurochir (Wien) 139:1061-1065, 1997

7. Kutz JW, Barnett SL, Hatanpaa KJ, Mendelsohn DB: Concurrent vestibular schwannoma and meningioma mimicking a single cerebellopontine angle tumor. Skull Base 19:443446, 2009

8. Nakamizo A, Suzuki SO, Shimogawa T, Amano T, Mizo- 
guchi $\mathrm{M}$, Yoshimoto $\mathrm{K}$, et al: Concurrent spinal nerve root schwannoma and meningioma mimicking single-component schwannoma. Neuropathology 32:190-195, 2012

9. Nakamura M, Tsuji O, Fujiyoshi K, Hosogane N, Watanabe $\mathrm{K}$, Tsuji T, et al: Long-term surgical outcomes of spinal meningiomas. Spine (Phila Pa 1976) 37:E617-E623, 2012

10. Ozawa H, Kokubun S, Aizawa T, Hoshikawa T, Kawahara C: Spinal dumbbell tumors: an analysis of a series of 118 cases. J Neurosurg Spine 7:587-593, 2007

11. Perry A, Lusis EA, Gutmann DH: Meningothelial hyperplasia: a detailed clinicopathologic, immunohistochemical and genetic study of 11 cases. Brain Pathol 15:109-115, 2005

12. Shuangshoti S, Netsky MG, Jane JA: Neoplasms of mixed mesenchymal and neuroepithelial type. With consideration of the relationship between meningioma and neurilemmoma. J Neurol Sci 14:277-291, 1971

13. Spencer WH: Diagnostic modalities and natural behavior of optic nerve gliomas. Ophthalmology 86:881-885, 1979

14. Zimmerman LE: Arachnoid hyperplasia in optic nerve glioma. Br J Ophthalmol 64:638-640, 1980

\section{Disclosure}

The authors report no conflict of interest concerning the materials or methods used in this study or the findings specified in this paper.

\section{Author Contributions}

Conception and design: Chikuda, Oichi. Acquisition of data: Oichi, Morikawa, Mori, Kitamura, Higuchi. Analysis and interpretation of data: Oichi, Morikawa, Mori, Taniguchi, Matsubayashi, Oshima. Drafting the article: Oichi. Critically revising the article: Chikuda, Morikawa. Reviewed submitted version of manuscript: Chikuda, Morikawa, Mori, Taniguchi, Matsubayashi, Oshima, Tanaka. Approved the final version of the manuscript on behalf of all authors: Chikuda. Study supervision: Chikuda, Tanaka.

\section{Correspondence}

Hirotaka Chikuda, Department of Orthopedic Surgery, The University of Tokyo Hospital, 7-3-1 Hongo, Bunkyo-ku, Tokyo 1138655, Japan. email: chikuda-tky@umin.ac.jp. 